\title{
Centros y servicios de información y documentación en la administración autonómica de Andalucía.
}

\author{
Situación actual
}

\author{
Jesús Gómez Fernández-Cabrera \\ Servicio de Documentación, Archivo y \\ Publicaciones. Consejería de Gobernación. \\ Junta de Andalucía \\ Inmaculada Maesso López \\ Unidad de documentación. \\ Gabinete de Análisis \\ y Relaciones Institucionales. \\ Presidencia de la \\ Junta de Andalucía
}

\section{INTRODUCCIÓN}

Conocer cuáles son los centros y servicios de documentación e información que existen en la Junta de Andalucía es una necesidad sentida por los profesionales del sector, e incluso por otros profesionales y por determinadas organizaciones públicas y privadas. Esta necesidad nos ha movido a publicar algunos resultados de una investigación llevada a cabo con objetivos más amplios, para dar a conocer qué centros y servicios existen en el aparato administrativo de la Junta de Andalucía y cuál es el perfil de estas unidades (especialización, recursos, etc).

Aquí presentamos un informe que recoge los datos relativos a determinados campos de nuestra investigación. Se trata de una foto fija en la que intentamos reflejar, de forma detallada, determinados aspectos de la gestión de la información y la documentación en el seno de la Administración autonómica andaluza.

Los datos se exponen de forma extructurada, acompañados de los comentarios imprescindibles para su comprensión. Hemos evitado expresar nuestra valoración, que ya expusimos en la presentación de la investigación hecha en las $V$ Jornadas Españolas de Documentación Automatizada', limitandonos aquí a un trabajo meramente expositivo, ofreciendo una herramienta que pensamos puede ser útil para conocer la realidad.

El informe se compone de tres capítulos y dos anexos: En el capítulo I, se presenta un mapa de las uni- dades documentales (UD) que existen en la Junta de Andalucía. En el capítulo 2 se presentan datos que pretenden dibujar un perfil descriptivo de dichas UD (antigüedad, cobertura temática, recursos personales, actividades y servicios documentales, automatización, etc). En el capítulo 3 los responsables de estas UD exponen su grado de satisfacción respecto a los distintos aspectos que son objeto de este estudio, aportando una información complementaria y valiosa para conocer la situación de las UD.

El Anexo / contiene una relación de las UD de la Junta de Andalucía, con las acotaciones necesarias para conocer de qué organismo dependen, tipo de adscripción, clase de UD, localidad, etc. En el Anexo 2 se describe la muestra de esta investigación, es decir se detallan las UD que han aportado sus informaciones a nuestra investigación?

La investigación está cerrada al 31 de mayo de 1996.

\section{UNIDADES DOCUMENTALES EN LA JUNTA DE ANDALUCÍA}

\section{Organismos de la Junta de Andalucía.}

Con el objeto de conocer las UD existentes en la Junta deAndalucía, consideramos que es necesario dibujar de forma previa el mapa del espacio organizativo sobre el que éstas se asientan:

\begin{tabular}{llr}
\hline \multirow{2}{*}{ Nombre de la Institución } & $\begin{array}{r}N^{0} \text { de organismos } \\
\text { existentes }\end{array}$ \\
\hline Parlamento de Andalucía & 1 \\
\hline \multirow{3}{*}{ Administración } & Consejerías y Presidencia & 13 \\
\cline { 2 - 3 } & Otros organismos & 56 \\
\cline { 2 - 3 } & Consejo Consult. de Andalucía & 1 \\
\hline Tribunal Sup. & Sede Granada & 1 \\
\cline { 2 - 3 } de Justicia & Sede Málaga & 1 \\
\cline { 2 - 3 } & Sala de Sevilla & 14 \\
\hline TOTAL & & 74 \\
\hline
\end{tabular}

Fig. I.- Organismos de la J.A. 
Estos son los organismos existentes en la Junta de Andalucía al 31 de mayo de 1996. Hay que hacer notar que el epígrafe "Otros organismos" engloba una serie de entes administrativos (organismos autónomos, empresas públicas, etc) difíciles de definir a veces y difíciles de localizar, lo que nos ha obligado a llevar a cabo una seleccion. En el Anexo I se relacionan estos organismos, y se establece una clasificación de los mismo.

\section{Unidades documentales existentes}

Después de consultar las fuentes publicadas 3 y de analizar la Relación de Puestos de Trabajo de la Junta de Andalucía hemos detectato la existencia de las siguientes UD:

\begin{tabular}{lrr}
\hline Organismos & $N^{0}$ de UD & $\%$ \\
\hline Parlamento de Andalucía & 4 & 6,34 \\
\hline Consejerías y Presidencia & 18 & 28,57 \\
\hline Otros organismos & 34 & 53,96 \\
\hline Consejo Consultivo de Andalucía & 1 & 1,58 \\
\hline Tribunal Sup. de Justicia de Andalucía & 6 & 9,52 \\
\hline TOTAL & 63 & 100,00 \\
\hline
\end{tabular}

Fig. 2.- UD existentes en los organismos

Conviene hacer una serie de acotaciones terminológicas y metodológicas, para exponer determinadas opciones que se han tomado al plantear la investigación.

Entendemos por UD aquella institución documental atendida por especialistas en información y documentación que tiene por objeto la recogida, análisis y tratatamiento de la información generada por fuentes internas y lo externas a la organización de la que forma parte; para lo que emplea medios personales, recursos técnicos y económicos, al objeto de someter la información a un tratamiento técnico documental que crea utilidad (económica o social) para los usuarios a quienes pretende satisfacer ${ }^{4}$. Hemos excluido en nuestro trabajo, como objeto de estudio los archivos y los museos, y nos hemos restringido al ámbito de las UD existentes en el aparato administrativo de la Junta de Andalucía, excluyendo tambien las bibliotecas públicas, las UD hospitalarias, etc.

\section{Distribución de las UD por consejerías}

\begin{tabular}{ll}
\hline Consejería & N $^{0}$ de UD \\
\hline Presidencia de la J.A. & 1 \\
\hline Consejería de la Presidencia & 3 \\
\hline Consejería de Gobernación & 1 \\
\hline Consejería de Economía y Hacienda & 1 \\
\hline Consejería de Relaciones con el Parlamento & 1 \\
\hline
\end{tabular}

\begin{tabular}{ll} 
Consejería de Trabajo e Industria & 2 \\
\hline Consejería de Turismo y Deportes & 2 \\
\hline Consejería de Obras públicas y Transportes & 1 \\
\hline Consejería de Agricultura y Pesca & 1 \\
\hline Consejería de Salud & 1 \\
\hline Consejería de Educación y Ciencia & 1 \\
\hline Consejería de Cultura & 2 \\
\hline Consejería de Medio Ambiente & 1 \\
\hline TOTAL & 18 \\
\hline
\end{tabular}

Fig. 3.- Distribución UD/consejerías

\section{Distribución de las UD en otros organismos}

Son muy diversos los organismos administrativos en cuanto a su definición júridica. La clasificación que aquí hacemos tiene una finalidad operativa. A continuación indicamos la existencia de UD en cada uno de estos tipos de centros:

\begin{tabular}{lccc}
\hline \multicolumn{1}{c}{ Organismo } & & Con UD & Sin UD \\
\cline { 1 - 2 } Tipo & $N^{0}$ & & \\
\hline Empresas públicas & 12 & 4 & 8 \\
\hline Organismos Autónomos & 9 & 6 & 3 \\
\hline Órganos de participación & 2 & 0 & 2 \\
\hline Servicios Administrativos & 22 & 13 & 9 \\
\hline Órganos periféricos & 11 & 11 & 0 \\
\hline TOTAL 56 & & 34 & 22 \\
\hline$\%$ & & 60,71 & 39,28 \\
\hline
\end{tabular}

Fig. 4.- Distribución UD/organismos

En el Anexo I puede identificarse la pertenencia de cada UD a su respectivo organismo, de forma individualizada.

\section{Distribución geográficas de las UD}

La ubicación territorial de las UD, se refleja en la siguiente tabla:

\begin{tabular}{lcccccccrr}
\hline & Almería & Cádiz & Córdoba & Granada & Huelva & Jaén & Málaga & Sevilla & Total \\
\hline Parlamento & & & & & & & 4 & 4 \\
\hline Consejerías & & & & & & & & 18 & 18 \\
\hline Otros org. & 2 & 3 & 2 & 6 & 1 & 1 & 3 & 16 & 34 \\
\hline C.Consult. & & & & 1 & & & & & 1 \\
\hline TS Justicia & & & & 1 & & & 2 & 3 & 6 \\
\hline TOTAL & 2 & 3 & 2 & 8 & 1 & 1 & 5 & 41 & 63 \\
\hline$\%$ & 3,17 & 4,76 & 3,17 & 12,69 & 1,58 & 1,58 & 7,93 & 65,07 & \\
\hline
\end{tabular}

Fig. 5.- Distribución geográfica 
Tipología de las UD

\begin{tabular}{lcrrr}
\hline \multirow{2}{*}{ Organismo } & \multicolumn{2}{c}{ Centro de Doc. } & \multicolumn{2}{c}{ Servicio de Doc. } \\
\cline { 2 - 5 } & No de UD & $\%$ & No de UD & $\%$ \\
\hline Parlamento de Andalucía & 0 & 0,00 & 4 & 6,34 \\
\hline Consejerías y Presidencia & 3 & 5,26 & 15 & 23,80 \\
\hline Otros organismos & 10 & 15,87 & 24 & 38,09 \\
\hline Consejo Consultivo de Andalucía & 0 & 0,00 & 1 & 1,58 \\
\hline Tribunal Superior de Justicia Andalucía & 0 & 0,00 & 6 & 9,52 \\
\hline TOTAL & 13 & 20,63 & 50 & 79,36 \\
\hline
\end{tabular}

Fig. 6.-Tipos de UD

\begin{tabular}{|c|c|}
\hline Año & $\mathrm{N}^{0}$ de UD \\
\hline 1909 & 1 \\
\hline 1975 & 4 \\
\hline 1976 & 0 \\
\hline 1977 & 0 \\
\hline 1978 & 1 \\
\hline 1979 & 0 \\
\hline 1980 & 5 \\
\hline 1981 & 0 \\
\hline 1982 & 0 \\
\hline 1983 & 1 \\
\hline 1984 & 3 \\
\hline 1985 & 0 \\
\hline 1986 & 4 \\
\hline 1987 & 5 \\
\hline 1988 & 4 \\
\hline 1989 & 2 \\
\hline 1990 & 4 \\
\hline 1991 & 2 \\
\hline 1993 & 4 \\
\hline 1995 & 0 \\
\hline 1996 & 0 \\
\hline $\mathrm{N} / \mathrm{C}$ & 2 \\
\hline TOTAL & 44 \\
\hline
\end{tabular}

Fig.7 Antigüedad de las UD
Por exigencia metodológica hemos establecido la distinción de dos tipos de UD: Servicios de Documentación y Centros de Documentación 5 . Hablaremos de Servicios para referirnos a las UD que integradas en un organismo administrativo prestan servicios documentales preferentemente a ese organismo, y hablaremos de Centros para referirnos a UD que, teniendo entidad organizativa propia o no, proyectan su actividad de forma horizontal en el conjunto de la Junta de Andalucía y en determinados sectores de la sociedad. (Ver Anexo I).

En la Junta de Andalucía, como en el resto de la Administración Pública, existen dos tipos de organismos: departamentos sectoriales con funciones de gestión, y organismos de tipo horizontal con funciones de estudio y apoyo técnico en áreas temáticas concretas. En cada una de estas instancias se genera y utiliza información y documentación, en parte común a todos los organismos (normativa jurídica, jurisprudencia, información de la Junta de Andalucía, etc.), y en parte diferenciada, en función de las actividades que le son propias a cada uno de ellos. Las Secretarías Generales Técnicas de estos departamentos tienen funciones ${ }^{6}$ tales como realizar estudios y reunir documentación sobre las materias propias del Departamento, preparar compilaciones de las disposiciones vigentes, cuidar de las publicaciones técnicas, dirigir y facilitar la formación de estadísticas, y otras más genéricas como mejorar la organización y métodos de trabajo y coordinación de los servicios. En consecuencia, cada organismo, que es responsable individualmente de la gestión de la información y documentación que necesita y/o produce, se ha ido dotando, a lo largo del tiempo, de los recursos organizativos, humanos y tecnológicos que dentro de sus posibilidades cada uno ha creído oportuno. Es decir, han ido creando lo que aquíllamamos Servicios de Documentación.

Respecto al otro tipo de instituciones documentales, los Centros de Documentación, decir que desde instancias distintas la Administración se han ido creando centros y sistemas con una definición documental clara y definida, cuando ha surgido la necesidad de conservar, tratar y/o servir documentación e información especializada. Así han surgido organismos como el Centro de Documentación Musical, Instituto de Estadística de Andalucía, Filmoteca de Andalu- cía, etc. Existen también centros que estando incardinados en un organismo superior, están diseñados para dar servicio más allá del organismo al que pertenecen: Instituto Cartográfico, Centro de Documentación del Patrimonio Histórico, Biblioteca de la Escuela de Salud Pública, Departamento de Documentación del Instituto Andaluz del Deporte, Centro de Documentación Juvenil, Oficinas de Información Administrativa al Ciudadano, Centro Servidor de Videotex de la Junta de Andalucía, Centro de Información y Documentación de la Junta de Andalucía (CIDJA), que desde la Consejería de la Presidencia produce la Base de Datos del BOJA, etc.

En la fig. 6 presentamos la cuantificación de Centros y Servicios de Documentación existentes en la Junta de Andalucía. En el Anexo I pueden identificarse cada uno de ellos de forma individualizada.

\section{PERFIL DE LAS UNIDADES DOCUMENTALES DE LA JUNTA DE ANDALUCÍA}

A continuación se aportan una serie de datos que pueden ser un instrumento para perfilar una imágen de cómo son las UD de la Junta de Andalucía.

\section{Antigüedad de las UD}

Se refleja aquí el año de creación de las distintas UD. Exepto la Biblioteca del Archivo de la Real Chancillería de Granada, creada en 1909 y transferida por el Estado, el resto de las UD son de creación autonómica: ver fig. 7

\section{Cobertura temática}

Las materias de que se ocupan las distintas UD de la Junta de Andalucía, y en qué medida, se reflejan en la siguiente tabla ${ }^{8}$.

\begin{tabular}{lcc}
\hline Temática & NNo de UD $^{0}$ & $\%$ \\
\hline 11. Lógica & 1 & 2,27 \\
\hline 12. Matemáticas & 2 & 4,54 \\
\hline 21. Astronomía y astrofísica & 1 & 2,27 \\
\hline 22. Física & 1 & 2,27 \\
\hline 23. Química & 12 & 27,27 \\
\hline 24. Ciencias de la vida & 3 & 6,81 \\
\hline 25. Ciencias de la Tierra y del Espacio & 5 & 11,36 \\
\hline 31. Ciencias Agronómicas & 12 & 27,27 \\
\hline 32. Ciencias Médicas & 7 & 15,90 \\
\hline 33. Ciencias Tecnológicas & 8 & 18,18 \\
\hline 51. Antopología & 6 & 13,63 \\
\hline 52. Demografía & 1 & 2,27 \\
\hline 53. Ciencias Económicas & 5 & 11,36 \\
\hline
\end{tabular}




\begin{tabular}{lcc} 
54. Geografía & 1 & 2,27 \\
\hline 55. Historia & 4 & 9,09 \\
\hline 56. Ciencias Jurídicas. Leyes & 19 & 43,18 \\
\hline 57. Lingüística & 1 & 2,27 \\
\hline 58. Pedagogía & 3 & 6,81 \\
\hline 59. Ciencia Política & 9 & 20,45 \\
\hline 61. Psicología & 2 & 4,54 \\
\hline 62. Ciencias del Arte. Letras & 6 & 13,63 \\
\hline 63. Sociología & 4 & 9,09 \\
\hline 71. Ética & 2 & 4,54 \\
\hline 72. Filosofía & 1 & 2,27 \\
\hline
\end{tabular}

Fig. 8.- Cobertura temática

\section{Cuantificación de los fondos 9}

\begin{tabular}{lrr}
\hline Tipo de documento & Total & Media \\
\hline Monografías & \multicolumn{1}{c}{260.827} & $6.520,67$ \\
\hline Manuales Técnicos & \multicolumn{1}{c}{4.059} & 101,47 \\
\hline Tesis, Proyectos fin de carrera ... & \multicolumn{1}{c}{917,00} & 22,92 \\
\hline Directorios, Obras de referencia ... & $3.578,00$ & 89,45 \\
\hline Informes de investigación, separatas & $35.235,00$ & 880,87 \\
\hline Revistas técnicas y científicas & $79.632,00$ & $1.990,80$ \\
\hline Revistas de resúmenes & 15,00 & 0,37 \\
\hline Boletines bibliográficos & 175,00 & 4,37 \\
\hline Boletines, Newsleetters ... & 156,00 & 3,90 \\
\hline Normas & 16,00 & 0,40 \\
\hline Mapas, planos, fotografías aéreas & $123.077,00$ & $3.076,92$ \\
\hline Actas de congresos & $2.508,00$ & 62,70 \\
\hline Películas, vídeos, ... & $7.181,00$ & 179,52 \\
\hline Diapositivas & $62.004,00$ & $1.550,10$ \\
\hline Microformas & $163.052,00$ & $4.076,30$ \\
\hline Discos y cassetes & $23.142,00$ & 578,55 \\
\hline CD-ROM & 146,00 & 3,65 \\
\hline Folletos & $6.392,00$ & 159,80 \\
\hline Memorias de empresas & 611,00 & 15,27 \\
\hline Recortes de prensa & $3.700,00$ & 92,50 \\
\hline Grabados y dibujos & 206,00 & 5,15 \\
\hline Diarios y revistas de información & 188,00 & 4,70 \\
\hline Piezas musicales (partituras) & $22.305,00$ & 557,62 \\
\hline Programas de ordenador & 262,00 & 6,55 \\
\hline Doc. de organismos internacionales & 120,00 & 3,00 \\
\hline Registros lexicológicos & & 31,17 \\
\hline Colecciones especiales & \\
\hline
\end{tabular}

Fig. 9.- Fondos

\section{Dotación de los recursos humanos}

La disponibilidad de efectivos personales y la cualificación de los mismos, es uno de los exponentes que pueden ayudarnos a saber la embergadura y la proyección de cualquier organización. Se reflejan a continuación algunos parámetos sobre los puestos de trabajo que existen en las UD objeto de nuestro estudio.

\section{CUANTIFICACIÓN DE LOS RECURSOS HUMANOS}

Los distintos tipos de puestos de trabajo que aparencen en la Relación de Puestos de Trabajo de la Junta de Andalucía podemos clasificarlos, haciendo un esfuerzo de abstracción y con el riesgo de simplificar, en tres categorias: a) Jefaturas de Servicio y Asesorías Técnicas (titulación superior); b) Jefaturas de Departamento o Sección (Diplomatura /Bachillerato); c) Jefatura de Negociado (Bachillerato /Graduado escolar) y d) Administrativo y Auxiliar Administrativo (Graduado escolar).

Siguiendo esta clasificación, en la siguiente tabla, se refleja la dotación de personal con que cuentan la UD.

\begin{tabular}{lccc}
\hline Tipo de Personal & Dotación total & Dotacion media & $\%$ \\
\hline Servicio/Asesores & 60 & 1,53 & 41,66 \\
\hline Departameto/sección & 19 & 0,48 & 13,19 \\
\hline Negociado/ Auxiliar & 65 & 1,66 & 45,13 \\
\hline TOTAL & 144 & 3,69 & \\
\hline
\end{tabular}

Fig. 10.- Dotación de personal

\section{TIPOLOGÍA LABORAL DE LOS PUESTOS DE TRABAJO}

El tipo de ocupación de las plazas por parte del empleado público que la ocupa, también es definitorio del perfil de la UD

\begin{tabular}{lcccccc}
\hline \multirow{2}{*}{$\begin{array}{l}\text { Tipos de } \\
\text { puestos de trabajo }\end{array}$} & \multicolumn{2}{c}{ Funcionario } & \multicolumn{2}{c}{ Interino } & \multicolumn{2}{c}{ Laboral } \\
\cline { 2 - 7 } & $\mathrm{N}^{0}$ & \multicolumn{1}{c}{$\%$} & $\mathrm{~N}^{0}$ & $\%$ & $\mathrm{~N}^{0}$ & $\%$ \\
\hline Servicio/Asesores & 24 & 16,66 & 13 & 9,02 & 23 & 15,97 \\
\hline Departameto/sección & 13 & 9,02 & 3 & 2,08 & 3 & 2,08 \\
\hline & 36 & 25,00 & 12 & 8,33 & 17 & 11,80 \\
\hline TOTAL & 73 & 50,69 & 28 & 19,44 & 43 & 29,89 \\
\hline
\end{tabular}

Fig. II.- Tipología de los puestos de trabajo

\section{DEFINICIÓN DOCUMENTAL DE LOS PUESTOS DE TRABAJO}

Es muy importante la cualificación profesional que se exige para ocupar los distintos puestos de trabajo. Bien por el nombre del propio puesto de trabajo, o bien porque se exijan conocimientos de Docu- 
mentación para su ocupación, hemos establecido la siguiente clasificación de los puestos de trabajo existentes: Puestos definidos como documentales y puestos, que realizando trabajos documentales, no lo refleja en su definición y por tanto no requieren para ser ocupados cualificación documental.

\begin{tabular}{lrrrr}
\hline & \multicolumn{2}{c}{$\begin{array}{c}\text { Definición } \\
\text { documental }\end{array}$} & \multicolumn{2}{c}{$\begin{array}{l}\text { Otras } \\
\text { definiciones }\end{array}$} \\
\hline Nervicio/Asesores & 51 & 35,41 & 9 & 6,25 \\
\hline Departameto/sección & 17 & 11,80 & 2 & 1,38 \\
\hline Negociado/ Auxiliar & 10 & 6,94 & 55 & 38,19 \\
\hline TOTAL & 78 & 54,16 & 66 & 45,83 \\
\hline
\end{tabular}

Fig. I2.- Definición documental de los puestos de trabajo

\section{Servicios documentales}

\section{TIPOS DE SERVICOS QUE PRESTAN LAS UD.}

En el siguiente cuadro se indican los distintos tipos de servicios documentales y el número de UD que prestan cada uno de ellos, con expresión de su correspondiente porcentaje:

\section{ÁMBITO DE LA PRESTACIÓN DE SERVICIOS}

\begin{tabular}{lcc}
\hline Tipo de servicio & N $^{0}$ de UD & $\%$ \\
\hline Sala de lectura & 33 & 75,00 \\
\hline Préstamo personal & 34 & 77,27 \\
\hline Préstamo interbibliotecario & 19 & 43,18 \\
\hline Reprografía de fondos propios & 36 & 81,81 \\
\hline Búsquedas retrospectivas & 29 & 65,90 \\
\hline Difusión selectiva de la informac. & 18 & 40,90 \\
\hline Cursos o seminarios & 9 & 20,45 \\
\hline
\end{tabular}

Fig. I3.- Servicios de la UD

\section{ACCESIBILIDAD}

Se pretende, con la siguiente tabla exponer el ámbito de actuación, en cuanto a prestación de servicios, de las UD. Se indica el número y el porcentaje de centros que presta cada servicio.

\begin{tabular}{lcc}
\hline Nivel de acceso & No $^{0}$ de UD & $\%$ \\
\hline Servicio/acceso al público & 24 & 54,54 \\
\hline Servicio/acceso restringido a organismos de la Administración Pública & 10 & 22,72 \\
\hline Servicio/acceso restringido a organismos de la Junta de Andalucía & 11 & 25,00 \\
\hline Servicio/acceso restringido a la propia Consejería/Organismo Aut./Empresa & 17 & 38,63 \\
\hline Servicio/acceso exclusivo a la Unidad administrativa a la que pertenece & 8 & 18,18 \\
\hline Investigadores acreditados & 3 & 6,81 \\
\hline
\end{tabular}

Fig. 14.- Accesibilidad de las UD
Hay que hacer notar, que los que hemos denominado Servicios de Documentación ${ }^{14}$, cuya vocación es servir a la institución a la que pertenecen, también prestan servicio a otras instituciones e incluso al público en general.

\section{Relaciones de cooperación}

La incorparación de las UD a actividades y organizaciones de cooperación queda reflejada a continuación:

\begin{tabular}{lcc}
\hline Tipo de cooperación & No $^{0}$ de UD & $\%$ \\
\hline Cooperan con otras organizaciones & 21 & 47,72 \\
\hline Son miembros de una organización & 10 & 22,72 \\
\hline Participan en Catálogos colectivos & 8 & 18,18 \\
\hline
\end{tabular}

Fig. 15.- Cooperación en las UD

\section{Recursos tecnológicos}

\section{ORDENADORES}

Para conocer el parque de ordenadores con que cuentan las UD hemos analizado en primer lugar el tipo de ordenador (grande/mini/micro) de que disponen.

\begin{tabular}{|c|c|c|c|c|c|}
\hline \multicolumn{2}{|c|}{ Grande } & \multicolumn{2}{|c|}{ Mini } & \multicolumn{2}{|c|}{ Micro } \\
\hline $\mathrm{N}^{0}$ & Media & $\mathrm{N}^{0}$ & Media & $\mathrm{N}^{0}$ & Media \\
\hline 0 & 0,00 & 3 & 0,06 & 84 & 1,90 \\
\hline
\end{tabular}

Fig. 16.- Tipos de ordenador

A continuación se desglosa el número de micro-ordenadores existentes:

\begin{tabular}{|c|c|c|}
\hline Tipo & $\mathrm{N}^{0}$ ordenadores & $\%$ \\
\hline Pentium & 4 & 4,76 \\
\hline 486 & 49 & 58,33 \\
\hline 386 & 17 & 20,23 \\
\hline 286 & 14 & 16,66 \\
\hline Total & 84 & 100,00 \\
\hline
\end{tabular}

Fig. 17.- Tipos de micro

\section{SOFTWARE}

En este apartado pretendemos conocer qué programas informáticos se utilizan en las UD y para que actividades se emplean.

Cuantificación de los programas utilizados

A continuación se indican los programas utilizados, con expresión del número y \% de UD donde se emplean. Hay que hacer notar que en una misma UD puede emplearse más de un programa. 


\begin{tabular}{lcc}
\hline Programa & N $^{0}$ de UD & $\%$ \\
\hline ABSYS & 7 & 11,86 \\
\hline ACCES MICROSOFF & 3 & 5,08 \\
\hline BASIS PLUS & 1 & 1,69 \\
\hline BEAT & 1 & 1,69 \\
\hline BRS & 2 & 3,38 \\
\hline CATDOC & 1 & 1,69 \\
\hline CDS-ISIS & 6 & 10,16 \\
\hline DBASE IV & 4 & 6,77 \\
\hline INMAGIL & 1 & 1,69 \\
\hline LIBERMAC & 1 & 1,69 \\
\hline KNOSYS & 16 & 27,11 \\
\hline NABIL & 3 & 5,08 \\
\hline OPEN ACCES & 1 & 1,69 \\
\hline PROCITE & 2 & 3,38 \\
\hline SABINI & 7 & 11,86 \\
\hline TINLIB & 1 & 1,69 \\
\hline TEXTO & 1 & 1,69 \\
\hline UNIPLEX & 1 & 1,69 \\
\hline Total instalaciones & 59 & \\
\hline & & \\
\hline
\end{tabular}

\begin{tabular}{lcc}
\hline Programa & N $^{0}$ de UD & $\%$ \\
\hline ABSYS & 7 & 15,90 \\
\hline CATDOC & 1 & 2,27 \\
\hline CDS-ISIS & 3 & 6,81 \\
\hline KNOSYS & 14 & 31,81 \\
\hline NABIL & 2 & 4,54 \\
\hline SABINI & 6 & 13,63 \\
\hline TEXTO & 1 & 2,27 \\
\hline & 34 & 77,27
\end{tabular}

Fig. 20.- Catalogación

\begin{tabular}{|c|c|c|}
\hline Programa & $\mathrm{N}^{0}$ de UD & $\%$ \\
\hline ABSYS & 4 & 9,09 \\
\hline CATDOC & 1 & 2,27 \\
\hline
\end{tabular}

Trabajos documentales implementados con cada programa En las cinco tablas siguientes se explica la utilización que se hace de cada uno de los programas. Se detallan las distintas actividades a que se aplican, con indicación del número y \% de UD que emplean ese program para esa actividad.

\begin{tabular}{lcc}
\hline CDS-ISIS & 1 & 2,27 \\
\hline LIBERMAC & 1 & 2,27 \\
\hline KNOSYS & 6 & 13,63 \\
\hline NABIL & 1 & 2,27 \\
\hline OPEN ACCES & 1 & 2,27 \\
\hline SABINI & 2 & 4,54 \\
\hline TINLIB & 1 & 2,27 \\
\hline TEXTO & 1 & 2,27 \\
\hline UNIPLEX & 1 & 2,27 \\
\hline & 20 & 45,45
\end{tabular}

Fig. 21.- Préstamo

\begin{tabular}{lcc}
\hline Programa & N $^{0}$ de UD & $\%$ \\
\hline ABSYS & 1 & 2,27 \\
\hline CATDOC & 1 & 2,27 \\
\hline KNOSYS & 4 & 9,09 \\
\hline OPEN ACCES & 1 & 2,28 \\
\hline & 7 & 15,90 \\
\hline
\end{tabular}

Fig. 22.- Gestión de publicaciones periódicas

\begin{tabular}{lcc}
\hline Programa & $N^{0}$ de UD & $\%$ \\
\hline ACCES & 2 & 4,54 \\
\hline BASIS PLUS & 1 & 2,27 \\
\hline BRS & 2 & 2,54 \\
\hline CDS-ISIS & 2 & 4,54 \\
\hline DBASE IV & 3 & 6,81 \\
\hline INMAGIL & 1 & 2,27 \\
\hline KNOSYS & 8 & 18,18 \\
\hline PROCITE & 1 & 2,27 \\
\hline & 20 & 45,45 \\
\hline
\end{tabular}

Fig. 23.- Bases de datos de elaboración propia 


\section{COMUNICACIONES}

A continuación se muestran los distintos elementos de telecomunicación con que tienen a su disposición las UD. Se expresa el número y porcentaje de UD que disponen de cada uno de ellos.

\begin{tabular}{lcc}
\hline & $N^{0}$ de UD & $\%$ \\
\hline Red telefónica básica & 44 & 100,00 \\
\hline RDSI & 2 & 4,54 \\
\hline X-25 & 13 & 29,54 \\
\hline Videotex & 13 & 29,54 \\
\hline Infovía & 4 & 9,09 \\
\hline Internet & 8 & 18,18 \\
\hline Proveedores de bases de datos & 11 & 25,00 \\
\hline Correo electrónico & 10 & 22,72 \\
\hline BBS & 6 & 13,66 \\
\hline Servidor Videotex & 2 & 4,54 \\
\hline Servidor Web & 1 & 2,27 \\
\hline
\end{tabular}

Fig. 24.- Comunicaciones y servicios a través de comunicaciones

\section{RED LOCAL}

La incorporación de las UD a la red local de su organización queda puesta de manifiesto en el siguiente cuadro, en el que se expresa el tipo de red, el número y porcentaje de UD que son usuarios de cada una.

\begin{tabular}{lcc}
\hline NOMBRE DELA RED & $\mathrm{N}^{0}$ de UD & $\%$ \\
\hline Banian & 1 & 2,27 \\
\hline Lan Smart & 1 & 2,27 \\
\hline Netware Novel & 12 & 27,27 \\
\hline Windows NT Server & 1 & 2,27 \\
\hline & 15 & 34,09 \\
\hline
\end{tabular}

Fig. 25.- Red local

\section{OTROS RECURSOS TECNOLÓGICOS}

La descripción del equipamiento tecnológico de las UD debemos completarla con el estudio de la dotación de otros recursos como los que se muestran en el siguiente cuadro, en el que se indica el número y porcentaje de UD que están dotados de cada uno de los recursos que se citan.

\begin{tabular}{lcc}
\hline & $N^{0}$ de UD & $\%$ \\
\hline Fax & 27 & 61,36 \\
\hline Scanner & 11 & 25,00 \\
\hline Lector microformas & 16 & 36,36 \\
\hline Fotocopiadora & 27 & 61,36 \\
\hline Lector CD-Rom & 15 & 34,09 \\
\hline Grabador CD-ROM & 5 & 11,36 \\
\hline OCR & 8 & 18,18 \\
\hline
\end{tabular}

Fig. 26.- Otros servicios tecnológicos

\section{Automatización. Utilización de los recursos tecnológicos}

Una vez que hemos puesto en claro los recursos tecnológicos de que disponen las UD, hemos de estudiar cuál es el empleo que de los mismos hacen. Para ello nos vamos a fijar en tres aspectos: actividades que se llevan a cabo de forma automatizada, cómo se realiza la catalogación de documentos y qué servicios y productos automatizados se producen.

\section{ACTIVIDADES AUTOMATIZADAS}

Se reseñan a continuación las actividades que las UD documentales realizan de forma automatizada, con indicación del número y porcentaje de UD que realizan cada una de las actividades.

\begin{tabular}{lcc}
\hline Actividades automatizadas & $\mathrm{N}^{0}$ de UD & $\%$ \\
\hline Adquisiciones & 15 & 34,09 \\
\hline Catalogación & 34 & 77,27 \\
\hline Control de suscripciones & 7 & 15,90 \\
\hline Préstamo & 20 & 45,45 \\
\hline Préstamo interbibliotecario & 6 & 13,63 \\
\hline Catálogo acceso público. OPAC & 7 & 15,90 \\
\hline Lenguaje documental:Tesauros... & 12 & 27,27 \\
\hline Difusión & 10 & 27,72 \\
\hline
\end{tabular}

Fig. 27.- Actividades automatizadas

\section{CATALOGACIÓN}

Buscamos ahora conocer cómo se lleva a cabo la catalogación en la actualidad en las distintas UD. Para ello se reseñan datos relativos a las normas empleadas en la catalogación, el nivel de profundidad de ésta, el formato de los registros, etc. Se indica siempre el número y porcentaje de UD que se encuentran en cada una de las situaciones que se describen.

\begin{tabular}{lcc}
\hline & $N^{0}$ de UD & $\%$ \\
\hline ISBD & 27 & 61,36 \\
\hline Normas propias & 10 & 22,72 \\
\hline N/C & 7 & 15,90 \\
\hline Total & 44 & \\
\hline
\end{tabular}

Fig. 28.- Normas de catalogación empleadas

\begin{tabular}{lcc}
\hline NIVEL & N $^{0}$ de UD & $\%$ \\
\hline Alto & 10 & 22,72 \\
\hline Medio & 20 & 45,45 \\
\hline Bajo & 8 & 18,18 \\
\hline N/C & 6 & 13,63
\end{tabular}

Fig. 29.- Catalogación. Nivel de profundidad 


\begin{tabular}{|c|c|c|}
\hline & $\mathrm{N}^{0}$ de UD & $\%$ \\
\hline Automatizada & 34 & 77,27 \\
\hline Manual & 10 & 22,72 \\
\hline
\end{tabular}

Fig. 30- Automatización de la catalogación

\begin{tabular}{lcc}
\hline & $\mathrm{N}^{0}$ de UD & $\%$ \\
\hline Ibermac & 22 & 68,75 \\
\hline Otros: & 10 & 31,25 \\
\hline N/C & 12 & \\
\hline
\end{tabular}

Fig. 3l.- Formato de los registros de entrada

\begin{tabular}{lcc}
\hline & N $^{0}$ de UD & $\%$ \\
\hline Ibermac & 17 & 38,63 \\
\hline Otros: & 7 & 15,90 \\
\hline N/C & 20 & 45,45 \\
\hline
\end{tabular}

Fig. 32.- Formato de los registros de salida

\begin{tabular}{lcc}
\hline & $\mathrm{N}^{0}$ de UD & $\%$ \\
\hline Bases de datos bibliográficas & 8 & 18,18 \\
\hline Bibliografías nacionales & 9 & 20,45 \\
\hline Catalogación propia & 36 & 81,81 \\
\hline Otras bibliotecas & 9 & 20,45 \\
\hline N/C & 3 & 6,81 \\
\hline
\end{tabular}

Fig .33.- Fuentes para la catalogación

\begin{tabular}{lcc}
\hline & $N^{0}$ de Ud & $\%$ \\
\hline Transferencia de ficheros & 5 & 11,36 \\
\hline Volcado registro a registro desde pantalla & 1 & 2,27 \\
\hline Tecleado a mano & 39 & 88,63 \\
\hline N/C & 5 & 11,36 \\
\hline
\end{tabular}

Fig. 34.- Mecanismo empleado en la catalogación

\begin{tabular}{lccc}
\hline Tipo de documento & Total & N $^{0}$ UD & Media \\
\hline Monografías & $125.189,00$ & 29 & 4316,86 \\
\hline Publicaciones periódicas (títulos) & 4199 & 15 & 279,93 \\
\hline Artículos de revista & $5.042,00$ & 3 & 1680,66 \\
\hline Disposiciones publicadas en BOJA & $128.658,00$ & 5 & 25731,60 \\
\hline Registros sonoros & $21.300,00$ & 1 & 21300,00 \\
\hline Vídeos / películas & $2.363,00$ & 3 & 787,66 \\
\hline Documentación gráfica & $140.950,00$ & 3 & 4698,30 \\
\hline Bienes Patrimonio Histórico & $31.790,00$ & 1 & 31790,00 \\
\hline Partituras & $22.000,00$ & 1 & 22000,00 \\
\hline
\end{tabular}

Fig. 35.- Registros automatizados

\section{SERVICIOS Y PRODUCTOS AUTOMATIZADOS}

Hay servicios que las UD prestan, en cuya ejecución se emplean en mayor o menor medida las tecnologías de la información. A continuación se mencionan esos servicios y se muestra el número y porcentaje de UD que los tienen automatizados.

\begin{tabular}{lcc}
\hline Servicios prestados & N $^{0}$ de UD & $\%$ \\
\hline Préstamo automatizado & 18 & 47,36 \\
\hline Préstamo con código de barras & 0 & 0,00 \\
\hline Acceso a bases de datos en CD-ROM & 15 & 39,47 \\
\hline Acceso a bases de datos en línea & 10 & 26,31 \\
\hline Boletín bibliográfico & 25 & 65,78 \\
\hline Obtención documentos primarios & 28 & 73,68 \\
\hline Boletín de resumenes & 19 & 50,00 \\
\hline Acceso a otros catálogos mediante OPAC & 5 & 13,15 \\
\hline Elaboración de informes/dossiers & 23 & 60,52 \\
\hline Acceso a Internet & 1 & 2,63 \\
\hline Acceso a Videtotex & 3 & 7,89 \\
\hline
\end{tabular}

Fig. 36.- Actividades/servicios automatizados

\section{AUTOVALORACIÓN DE LA DOTACIÓN DE RECURSOS DE LAS UD}

Hemos requerido a los responsables de las distintas UD que manifiesten su grado de satisfacción (alto, medio o bajo) acerca de las dotaciones con que cuentan sus respectivos centros y servicios. Estas opiniones se muestran en la tabla siguiente en la que se indica el número y porcentaje de las correspondientes a las UD que opinan sobre cada uno de los aspectos que se valoran.

\begin{tabular}{|c|c|c|c|c|c|c|c|c|}
\hline \multirow[t]{3}{*}{ Aspecto que se valora } & \multicolumn{8}{|c|}{ Grado de satisfacción } \\
\hline & \multicolumn{2}{|c|}{ Alto } & \multicolumn{2}{|c|}{ Medio } & \multicolumn{2}{|c|}{ Bajo } & \multicolumn{2}{|c|}{$\mathrm{N} / \mathrm{C}$} \\
\hline & $\mathrm{N}^{0}$ de UD & $\%$ & $\mathrm{~N}^{0}$ de UD & $\%$ & $\mathrm{~N}^{0}$ de UD & $\%$ & $\mathrm{~N}^{0}$ de UD & $\%$ \\
\hline$\overline{\text { Dotación de personal }}$ & 3 & 6,81 & 13 & 29,54 & 25 & 56,81 & 3 & 6,81 \\
\hline Recursos económicos & 4 & 9,09 & 18 & 40,90 & 19 & 43,18 & 3 & 6,81 \\
\hline Recursos materiales & 5 & 11,36 & 13 & 29,54 & 23 & 52,27 & 3 & 6,81 \\
\hline Ordenadores & 8 & 18,18 & 21 & 47,72 & 12 & 27,27 & 3 & 6,81 \\
\hline Programas informáticos & 10 & 22,72 & 15 & 34,09 & 16 & 36,36 & 3 & 6,81 \\
\hline Red local & 5 & 11,38 & 3 & 6,81 & 11 & 25,00 & 25 & 56,81 \\
\hline Comunicaciones & 6 & 13,63 & 6 & 13,63 & 24 & 54,54 & 8 & 18,18 \\
\hline
\end{tabular}

Fig. 37.- Autovaloración de los recursos 


\section{ANEX0 1:}

\section{CENTROS Y SERVICIOS DE DEOCUMENTACIÓN DE LA JUNTA DE ANDALUCIA Y MUESTRA DE LA INVESTIGACION}

\begin{tabular}{|c|c|c|c|c|}
\hline Organismo/Centro Directivo & Nombre de la UD & Adscripción & Tipo & Muestra \\
\hline \multicolumn{5}{|l|}{ PARLAMENTO } \\
\hline \multirow[t]{2}{*}{ Dirección General de Servicios } & Servicio de Documentación y Archivo & & & \\
\hline & C/. Andueza, s/n. .41009 SEVILLA. TF 4592100 & $P$ & S & NO \\
\hline \multirow[t]{2}{*}{ Dirección General de Servicios } & Servicio de Biblioteca & & & \\
\hline & C/. Andueza , s/n. .41009 SEVILLA. TF 4592100 & $P$ & S & $\mathrm{SI}$ \\
\hline \multirow[t]{3}{*}{ Cámara de Cuentas } & Servicio de Biblioteca y Archivo & & & \\
\hline & Av. Ramón y Cajal, 35. 41005 SEVILLA. & & & \\
\hline & TF 95-4662701 & $P$ & S & $\mathrm{SI}$ \\
\hline \multirow[t]{3}{*}{ Defensor del Pueblo } & Servicio de Documentación y Biblioteca & & & \\
\hline & C/ Reyes Católicos, 21. 41001 SEVILLA. & & & \\
\hline & TF $95-4212121$ & $P$ & S & $\mathrm{SI}$ \\
\hline \multicolumn{5}{|c|}{ TRIBUNAL SUPERIOR DE JUSTICIA DE ANDALUCÍA } \\
\hline \multirow[t]{3}{*}{$\bar{T}$ T.S.J } & Biblioteca & & & \\
\hline & Palacio de la Real Chancillería. PI. Nueva s/n. 18010. & & & \\
\hline & GRANADA. TF. 958-242108; 242109 & TS & $S$ & NO \\
\hline \multirow{2}{*}{ Audiencia Provincial } & Biblioteca & & & \\
\hline & Hotel Miramar. MÁLAGA. 95-2136425 & TS & S & NO \\
\hline \multirow[t]{2}{*}{ Sala de lo Contencioso Advo. } & Biblioteca & & & \\
\hline & Hotel Miramar. MÁLAGA. 95-2136425 & TS & S & NO \\
\hline \multirow[t]{2}{*}{ Sala de lo Contencioso Advo. } & Biblioteca & & & \\
\hline & Edificio Viapol. SEVILLA & TS & S & NO \\
\hline \multirow[t]{2}{*}{ Audiencia Provincial } & Biblioteca & & & \\
\hline & Avenida de Menéndez Pelayo, 2. SEVILLA & TS & $S$ & NO \\
\hline \multirow[t]{2}{*}{ Juzgados $1^{\mathrm{a}}$ Instancia } & Biblioteca & & & \\
\hline & Edificio Viapol. SEVILLA & TS & S & NO \\
\hline \multicolumn{5}{|l|}{ PRESIDENCIA DE LA J.A. } \\
\hline \multicolumn{5}{|l|}{ Gabinete de Análisis y } \\
\hline Relaciones Institucionales & Av. de Roma, s/n. 41013 SEVILLA. TF 4597524 & C & S & $\mathrm{SI}$ \\
\hline \multicolumn{5}{|c|}{ CONSEJERÍA DE LA PRESIDENCIA } \\
\hline \multirow[t]{2}{*}{ Secretaría General Técnica } & $\begin{array}{l}\text { Centro de Información y Documentación } \\
\text { de la Junta de Andalucía (CIDJA) }\end{array}$ & & & \\
\hline & C/ Alfonso XII, 17. 41002 SEVILLA. TF 95-4218860 & C & C & SI \\
\hline \multirow{3}{*}{$\begin{array}{l}\text { Dirección General de Asuntos } \\
\text { Europeos y Cooperación Exterior }\end{array}$} & Servicio de Relaciones Institucionales, & & & \\
\hline & Asesoramienrto e Información & & & \\
\hline & C/ Alfonso XII, 17.41002 SEVILLA. TF 95-4218860 & C & S & $\mathrm{SI}$ \\
\hline \multirow[t]{2}{*}{ Gabinete Jurídico } & Documentación & & & \\
\hline & C/ Alfonso XII, 17.41002 SEVILLA. TF 95-4218860 & c & S & NO \\
\hline \multirow{3}{*}{$\begin{array}{l}\text { Empresa Pública de Radio y } \\
\text { Televisión de Andalucía }\end{array}$} & Documentación & & & \\
\hline & Carretera de San Juan de Aznalfarache-Tomares, Km 1'3 & & & \\
\hline & $\begin{array}{l}41920 \text { SAN JUAN DE AZNALFARACHE } \\
\text { (SEVILLA). TF } 955607600\end{array}$ & EP & $S$ & NO \\
\hline \multirow[t]{2}{*}{ Instituto Andaluz de la Mujer } & Departamento de Documentación y Publicaciones & & & \\
\hline & C/ Alfonso XII, 52. 41002 SEVILLA. TF 95-4904800 & $O A$ & S & SI \\
\hline \multicolumn{5}{|l|}{ CONSEJERÍA DE GOBERNACIÓN } \\
\hline \multirow[t]{2}{*}{ Secretaría General Técnica } & Servicio de Documentación Archivo y Publicaciones & & & \\
\hline & PI. Nueva, 4. 41071 SEVILLA. TF 45575500. & C & S & $\mathrm{SI}$ \\
\hline
\end{tabular}


Instituto Andaluz de

Departamento de Estudios y Documentación

Administración Pública

C/ Mª Auxiliadora,13. 41071 SEVILLA. TF 95-4559602

CONSEJERÍA DE ECONOMÍA Y HACIENDA

\begin{tabular}{|c|c|c|c|c|}
\hline Viceconsejería & $\begin{array}{l}\text { Biblioteca } \\
\text { Av. República Argentina, 25, 8a plta. } \\
41011 \text { SEVILLA. TF 95-4555358 }\end{array}$ & C & S & SI \\
\hline \multirow{3}{*}{$\begin{array}{l}\text { Instituto de Estadística de } \\
\text { Andalucía }\end{array}$} & Biblioteca & & & \\
\hline & C/ Marqués de Nervión, 40. 41071 & & & \\
\hline & SEVILLA. TF 95- 4272426. & $\mathrm{OA}$ & S & NO \\
\hline \multicolumn{5}{|c|}{ CONSEJERÍA DE RELACIONES CON EL PARLAMENTO } \\
\hline \multirow[t]{2}{*}{ Secretaría General Técnica } & Sección de Coordinación y Documentación & & & \\
\hline & C/ Alfonso XII, 17. 41001 SEVILLA. TF 95-4218860 & C & $S$ & $\mathrm{SI}$ \\
\hline \multicolumn{5}{|c|}{ CONSEJERÍA DE TRABAJO E INDUSTRIA } \\
\hline \multirow[t]{2}{*}{ Secretaría General Técnica } & Biblioteca & & & \\
\hline & Avda. Héroes de Toledo, s/n . 41071 SEVILLA. TF 95-4555233. & C & $S$ & NO \\
\hline \multirow{3}{*}{$\begin{array}{l}\text { Instituto Andaluz de } \\
\text { Servicios Sociales }\end{array}$} & Departamento de Documentación & & & \\
\hline & Av. Héroes de Toledo, s/n. & & & \\
\hline & 41071 SEVILLA. TF 95-4554041 & $\mathrm{OA}$ & S & $\mathrm{SI}$ \\
\hline \multicolumn{5}{|l|}{ Instituto de Fomento de } \\
\hline Andalucía & No existe UD & EP & & NO \\
\hline \multicolumn{5}{|l|}{ Consejo Andaluz de } \\
\hline Relaciones Laborales & No existe UD & $\mathrm{OP}$ & & NO \\
\hline \multirow[t]{3}{*}{ Comisionado para la Droga } & Centro de Documentación & & & \\
\hline & Av. Héroes de Toledo, s/n & & & \\
\hline & 41071 SEVILLA. TF 95-4555100 & C & C & $\mathrm{SI}$ \\
\hline \multicolumn{5}{|c|}{ CONSEJERIA DE TURISMO Y DEPORTES } \\
\hline \multirow[t]{2}{*}{ Secretaría General Técnica } & Servicio de Legislación y Recursos. Biblioteca & & & \\
\hline & Avda. Héroes de Toledo, s/n . 41071 SEVILLA. & C & $S$ & $\mathrm{SI}$ \\
\hline \multirow[t]{3}{*}{ Dirección Genral de Turismo } & Servicio de Infraestructura & & & \\
\hline & Av. República Argentina, 25, 8ª plta. & & & \\
\hline & 41011 SEVILLA. TF 95-4558411 & C & S & $\mathrm{SI}$ \\
\hline \multirow[t]{3}{*}{ Instituto Andaluz del Deporte } & Departamento de Documentación y Publicaciones & & & \\
\hline & Av. Santa Rosa de Lima, 5. & & & \\
\hline & 29007 MALAGA. TF 95-22282266. & SA & C & $\mathrm{SI}$ \\
\hline Empresa Pública de Turismo & No existe UD & EP & & NO \\
\hline \multicolumn{5}{|c|}{ CONSEJERÍA DE OBRAS PÚBLICAS Y TRANSPORTES } \\
\hline \multirow[t]{2}{*}{ Secretaría General Técnica } & Biblioteca Central & & & \\
\hline & Av. de la Constitución , 25. 41001 SEVILLA.TF 95- 4219169. & C & S & $\mathrm{SI}$ \\
\hline \multirow[t]{2}{*}{ Empresa Publica del Suelo } & Archivo y Documentación & & & \\
\hline & Av. de Bonanza, 4, 2ª plta. 41012 SEVILLA. . TF 95- 4550292. & EP & S & $\mathrm{SI}$ \\
\hline Empresa Pública de Puertos & No existe UD & EP & & NO \\
\hline \multirow[t]{3}{*}{ Instituto de Cartografía de Andalucía } & Instituto de Cartografía de Andalucía & & & \\
\hline & Av. de la Constitución , 25. & & & \\
\hline & 41001 SEVILLA. TF 95-4218180. & SA & C & SI \\
\hline \multicolumn{5}{|c|}{ CONSEJERÍA DE AGRICULTURA Y PESCA } \\
\hline \multirow{3}{*}{$\begin{array}{l}\text { Dirección General de } \\
\text { Investigación y Formación Agraria }\end{array}$} & Biblioteca General & & & \\
\hline & C/ Juan de Lara Nieto s/n & & & \\
\hline & 41013 SEVILLA. TF 95- 4551800. & C & S & $\mathrm{SI}$ \\
\hline Centro de Investigación y & Biblioteca & & & \\
\hline Formación Agraria (CIFA). & Apartado 240. 14080 CÓRDOBA. TF 957-295539 & $\mathrm{CP}$ & $S$ & SI \\
\hline Centro de Investigación & Biblioteca & & & \\
\hline y Formación Agraria (CIFA). & Apartado 91. 04700 EL EJIDO ( ALMERÍA). & CPEL & $S$ & SI \\
\hline Centro de Investigación y & Biblioteca & & & \\
\hline Formación Agraria (CIFA) & Apartado 159. MÁLAGA. TF 95-2622640 & $\mathrm{CP}$ & S & SI \\
\hline
\end{tabular}


Centro de Investigación y Formación Agraria (CIFA)

Centro de Investigación y

Formación Agraria (CIFA)

Centro de Investigación y

Formación Agraria (CIFA)

Centro de Investigación de Cultivo
de Especies Marinas (CICEM)

de Especies Marinas (CICEM)

Centro de Investigación de Cultivo

de Especies Marinas (CICEM)

Centro de Investigación y

Formación Agraria (CIFA)

\begin{tabular}{l} 
Centro de Investigación y \\
Formación Agraria (CIFA) \\
\hline Empresa Andaluza de \\
Gestión de Tierras \\
\hline InstitutoAnd. de Reforma Agraria \\
\hline CONSEJERÍA DE SALUD \\
\hline Secretaría General Técnica
\end{tabular}

Servicio Andaluz de Salud

Biblioteca

Apartado de Correos Oficial

41200 ALCALÁ DEL RíO (SEVILLA)

\section{Biblioteca}

Apartado 2027 GRANADA. TF 958-267311

Biblioteca

Apartado 121. 410889 MONTEQUINTO (SEVILLA)

TF 954616511

Biblioteca

Apartado 104. 21450 CARTAYA ( HUELVA). TF 959-399104 CP $\quad$ S $\quad$ SI

Biblioteca

Apartado 16. EL PUERTO DE STA. Ma CÁDIZ. TF 956-562342 $\quad$ CP $\quad$ S $\quad$ SI

\section{Biblioteca}

Apartado 589. 11480 JEREZ DE LA FRONTERA (CÁDIZ).

TF 956-312570

Biblioteca

Apartado 50. 23620 MENGÍBAR ( JAÉN). TF 953-370150 CP $\quad$ S $\quad$ SI

Sección de Documentación y Publicaciones

Av. de la Constitución, 18. 41001 SEVILLA.

TF 95-4211602

OA S NO

Escuela Andaluza de Salud Pública

Biblioteca

Apartado 2070. Campus Universitaio de Cartuja, s/n.

18011 GRANADA. TF 161044.

EP $\quad$ C $\quad S I$

Agencia de Evaluación de

Tecnologías Sanitarias de Andalucía

Emp. Pública Hospital Costa del Sol

No existe UD

SA

Documentación

MARBELLA (MÁLAGA)

EP

$\mathrm{OA}$

NO

\begin{tabular}{llll} 
& MARBELLA (MÁLAGA) & EP & C NO \\
\hline Emp. Púb. de Emergencias Sanitarias & No existe UD & EP & NO \\
\hline
\end{tabular}

CONSEJERÍA DE EDUCACIÓN Y CIENCIA

Secretaría General Técnica

Sección de Documentación y Publicaciones. Biblioteca

Av. República Argentina, 21 B, $3^{\text {a }}$ plta. 41011

SEVILLA. TF 4558400

C S NO

Instituto Andaluz de

Energías Renovables

No existe UD

SA

NO

Instituto Andaluz de Evaluación

Educativa y Form. del Profesorado

Servicio Andaluz de Micrografía

No existe UD

SA

Instituto Andaluz Biotecnología

No existe UD

No existe UD

SA

Centro Andaluz de Prospectiva

No existe UD

SA

Centro de Informática

Biblioteca

Científica de Andalucía (CICA)

C/ Tarifa, s/n. Campus Reina Mercedes.

41071 SEVILLA. TF 4623811

SA S NO

Instituto Andaluz de Criminología

No existe UD

SA

Centro Andaluz de Documentación

en Normalización y Fabricación

No existe UD

SA

\begin{tabular}{llll} 
en Normalización y Fabricación & No existe UD & SA & NO \\
\hline Consejo Escolar de Andalucía & No existe UD & OP & NO
\end{tabular}




\begin{tabular}{|c|c|c|c|c|}
\hline \multicolumn{5}{|l|}{ CONSEJERÍA DE CULTURA } \\
\hline \multirow[t]{2}{*}{ Secretaría General Técnica } & \multicolumn{4}{|l|}{ Servicio de Estudios y Publicaciones } \\
\hline & C/ San josé, 13. 41071 SEVILLA. TF 95-4559999 & C & S & $\mathrm{Sl}$ \\
\hline \multicolumn{4}{|l|}{ Empresa Pública de Gestión } & NO \\
\hline Instituto Andaluz de & Centro de Documentación del Patrimonio Histórico & & & \\
\hline \multirow[t]{2}{*}{ Patrimonio Histórico } & Av. Isla de la Cartuja, 1. 41071 SEVILLA. & & & \\
\hline & TF 95-4461074. TF 4480650 & SA & C & SI \\
\hline \multicolumn{5}{|l|}{ Centro Andaluz de } \\
\hline Arte Contemporáneo & No existe UD & $\mathrm{OA}$ & & NO \\
\hline Conjunto Monumental Cartuja & Biblioteca & SA & S & NO \\
\hline Consejo de la Juventud de Andalucía & No existe UD & EP & & NO \\
\hline Centro de Documentación & Centro de Documentación Músical de Andalucía & & & \\
\hline Músical de Andalucía & Carrera del Darro, 29. 18010 GRANADA. TF 223610 & SA & C & $\mathrm{SI}$ \\
\hline \multirow[t]{3}{*}{ Centro Andaluz del Flamenco } & Biblioteca & & & \\
\hline & PI. San Juan, 1. 11403 JEREZ DE LA FRONTERA (CÁDIZ) & & & \\
\hline & TF 956-349265 & SA & & NO \\
\hline \multirow[t]{2}{*}{ Centro Andaluz del Teatro } & Unidad de Documentación & & & \\
\hline & C/ San Luis, 37. 41003 SEVILLA. TF 95-4901493 & SA & C & $\mathrm{SI}$ \\
\hline \multirow[t]{3}{*}{ Biblioteca de Andalucía } & Biblioteca de Andalucía & & & \\
\hline & C/ Profesor Sainz Cantero, 6. 18002 GRANADA. & & & \\
\hline & TF 958-282820 & SA & C & $\mathrm{Sl}$ \\
\hline \multirow[t]{2}{*}{ Archivo General de Andalucía } & Biblioteca Auxiliar & & & \\
\hline & C/Almirante Apodaca, 2. 41003 SEVILLA. TF 95-4218248 & SA & S & $\mathrm{SI}$ \\
\hline \multirow[t]{2}{*}{ Archivo de la Real Chancillería } & Biblioteca Auxiliar & & & \\
\hline & PI. del Padre Suárez, 1. 18009 GRANADA. TF 958-222338 & $\mathrm{CP}$ & S & $\mathrm{SI}$ \\
\hline \multicolumn{5}{|l|}{ Empresa Andaluza de Instalaciones } \\
\hline y Turismo Juvenil & No existe UD & $\mathrm{OA}$ & & NO \\
\hline Centro Andaluz de Danza & No existe UD & SA & & NO \\
\hline \multirow[t]{3}{*}{ Patronato de la Alhambra y Generalife } & Biblioteca y Archivo de la Alhambra & & & \\
\hline & Biblioteca y Archivo de la Alhambra. & & & \\
\hline & C/ Real s/n. 18009 GRANADA. TF 227525 & $\mathrm{OA}$ & S & $\mathrm{SI}$ \\
\hline \multirow[t]{2}{*}{ Centro Andaluz de la Fotografía } & Centro Andaluz de la Fotografía & & & \\
\hline & C/ Martinez Campos, 20. 04002 ALMERÍA. & SA & C & NO \\
\hline \multirow[t]{2}{*}{ Filmoteca de Andalucía } & Filmoteca de Andalucía & & & \\
\hline & C/ Medina y Corella, 5. 14003 CÓRDOBA. TF 957-481835 & SA & C & $\mathrm{SI}$ \\
\hline Escuela Pública de & Documentación & & & \\
\hline Animación Sociocultural & C/ Bilbao, 8-10. 41001 SEVILLA. TF 4223044/4222619 & SA & S & NO \\
\hline \multirow[t]{2}{*}{ D. G. de Juventud y Voluntariado } & Centro Andaluz de Documentación Juvenil & & & \\
\hline & C/ Muñoz Olivé, 1, 3ª plta. 41071 SEVILLA. TF 4226943 & C & C & NO \\
\hline \multicolumn{5}{|l|}{ CONSEJERIIA DE MEDIO AMBIENTE } \\
\hline \multirow[t]{2}{*}{ Secretaría Genral Técnica } & Biblioteca & & & \\
\hline & Av. Eritaña, 1. 41013 SEVILLA. TF 4550550 & C & S & $\mathrm{SI}$ \\
\hline Empresa de Gestión Medioambiental & No existe UD & EP & & NO \\
\hline \multicolumn{5}{|c|}{ CONSEJO CONSULTIVO DE ANDALUCÍA } \\
\hline \multirow[t]{2}{*}{ Secretaría General } & Unidad de Documentación & & & \\
\hline & C/ San Matías, 17. 18071 GRANADA. TF 958-221384 & CC & S & SI \\
\hline
\end{tabular}

\section{INTERPRETACIÓN DE LOS SIGNOS}

ADCRIPCIÓN: C: Consejería CC: Consejo Consultivo de Andalucía CP: Órgano periférico EP: Empresa Pública

OA: Organismo Autónomo OP: Órgano de participación con funciones administrativas. P: Parlamento de Andalucía

SA: Servicio Administrativo sin sin personalidad júridica propia

TS: Tribunal Superior de Justicia de Andalucía
TIPO DE UNIDAD DOCUMENTAL (Ver apartado 6.I)

C: Centro de Documentación S: Servicio de Documentación MUESTRA

SI: Esta UD sí está incluida en la muestra que ha servido de base al estudio

NO. Esta UD no está incluida en la muestra que ha servido de base al estudio 
ANEXO 2

\section{MUESTRA DE LA INVESTIGACIÓN}

Las UD que han aportado sus datos a la investigación, respondiendo a nuestro cuestionario, y a nuestra petición de aclaraciones, han sido las siguientes:

\begin{tabular}{lrr}
\hline Organismo & N $^{0}$ de UD & $\%$ \\
\hline Parlamento de Andalucía & 3 & 75,00 \\
\hline Consejerías y Presidencia & 15 & 83,33 \\
\hline Otros organismos & 25 & 73,52 \\
\hline Consejo Consultivo de Andalucía & 1 & 100,00 \\
\hline Tribunal Superior de Justicia A. & 0 & 0,00 \\
\hline TOTAL & 44 & 69,84 \\
\hline
\end{tabular}

Fig. 38. UD estudiadas. Muestra19

En el Anexo I pueden identificarse cada una de estas UD. Hay que hacer notar que algunas han quedado fuera de la muestra no por voluntad de sus responsables, sino por fallos imputables a los promotores de la investigación.
Conforme a la distinción que hemos establecido en el Apartado 1.6, en nuestra muestra conviven dos tipos de instituciones documentales, los Centros y los Servicios. Hemos optado por el tratamiento unitario de la información de ambos, aunque conscientes que en algunos aspectos las características de unos puedan distorsionar el perfil del conjunto. Para evitar este riesgo, los datos estadísticos se han elaborado teniendo en cuenta esta distinción en los casos que ha sido procedente.

\begin{tabular}{lcr}
\hline Tipo de UD & N$^{0}$ de UD & $\%$ \\
\hline Centro de Doc. & 10 & 22,72 \\
\hline Servicio de Doc & 34 & 77,27 \\
\hline Total & 44 & 100,00 \\
\hline
\end{tabular}

Fig. 39. tipologías de las UD de la muestra20

Notas

I. Esta investigación fué presentada en las $\vee$ Jornadas Españolas de Documentación Automatizada, celebradas en Cáceres de 17 al 19 de Octubre de 1996. Allí expusimos los objetivos de nuestro trabajo y las fases y metodología empleados, así como algunas de las conclusiones que de él pueden extraerse. Ver GÓMEZ, J. y MAESSO, l.: Gestión automatizada de la información en la Administración. En Sistemas de Información. Balance de 12 años de jornadas y perspectivas. V Jornadas Españolas de Documentación Automatizada. Actas. Cáceres: Fesabid, 1996, pp. 403-412

2. Queremos reflejar aquí nuestro sincero agradecimiento a todos los colegas que, aportando los datos de sus respectivos centros, han hecho posible este trabajo.

3. MARTíN OÑATE, A. (dir.): Directorio bibliotecario de Andalucía. Málaga: Asociación andaluza de bibliotecarios, 1995.

MINISTERIO DE CULTURA: Mapa de infraestructuras, operadores y recursos culturales (MIORC). Madrid: Ministerio de Cultura, 1995

OFICINA DEL PORTAVOZ DEL GOBIERNO: Agenda de la comunicación 1996. Sevilla: Consejería de la Presidencia, 1995

DIRECCIÓN GENERAL DE ORGANIZACIÓN Y

MÉTODOS E IGS: Guía de Funciones y Servicios de la

Administración Andaluza. Sevilla: Consejería de Gobernación, 1995

4. RAMOS SIMÓN, L. F.: Dirección, administración y marqueting de empresas e instituciones documentales. Madrid: Ed. Síntesis, 1995, p

5. Esta distinción está inspirada en la clasificación que propone J.. CHAUMIER en Técnicas de Documentación y Archivo. Barcelona: Oikos-Tau, 1993, pp. 25-28.

6. Ley 6/1983, de 21 de julio, de Gobierno y Administración de la Comunidad Autónoma. BOJA, 60, 29.07.83, art. 42.

7. El \% se refiere al número total de UD de la JA
8. Hemos seguido para la clasificación de los temas la Nomenclatura para los campos de las ciencias y la tecnología de la Unesco

9. A efectos de calcular la media de este ítem se ha computado la información de 40 UD

10. A efectos de calcular la media y el \% de este ítem se ha computado la información de 39 UD

I I. El \% hace referencia al total de personas que trabajan en UD: 144

12. Hay que hacer notar que aunque la denominación del pues to haga alusión a la Documentación, no por ello se exigen conocimientos específicos para su ocupación. En la mayoría de los casos la exigencia se refiere sólamente al nivel de titulación, no a la especialidad. En la actualidad se están implantando las Ilamadas "Areas Funcionales", que inciden en la especialización de experiencia requerida para ocupar los puestos.

13. El \% hace referencia al total de personas que trabajan en UD: 144

|4. Ver apartado I.6

|5. Para el cálculo del \% se han computado los datos de 4I UD.

16. Hay que hacer notar que en una misma UD se pueden emplear distintos menacismos.

17. Pretendemos mostrar aquí el volumen de registros que contienen los catálogos automatizados de las UD. No todos los centros disponen de los mismos tipos de documentos, por lo cual en la tercera columna se indica el número de centros que disponen de cada uno de los tipos documentales, siendo ese el número empleado para obtener la media.

18. Para la obtención del porcentaje se han computado los datos relativos a 38 UD

19. El \% se refiere al número total de UD existentes en la JA

20. El \% se refiere al número de UD que componen la muestra 